\title{
A Model of Strongly Correlated Electrons with Condensed Resonating-Valence-Bond Ground States
}

\author{
Akinori Tanaka ${ }^{1}$ \\ Department of Applied Quantum Physics, Kyushu University, Fukuoka 812-8581, Japan
}

(September 12, 2003)

\begin{abstract}
We propose a new exactly solvable model of strongly correlated electrons. The model is based on a $d-p$ model of the $\mathrm{CuO}_{2}$ plane with infinitely large repulsive interactions on $\mathrm{Cu}$-sites, and it contains additional correlated-hopping, pair-hopping and charge-charge interactions of electrons. For even numbers of electrons less than or equal to $2 / 3$-filling, we construct the exact ground states of the model, all of which have the same energy and each of which is the unique ground state for a fixed electron number. It is shown that these ground states are the resonating-valence-bond states which are also regarded as condensed states in which all electrons are in a single two-electron state. We also show that the ground states exhibit off-diagonal long-range order.
\end{abstract}

\footnotetext{
${ }^{1}$ t-akitap@mbox.nc.kyushu-u.ac.jp
} 


\section{Introduction}

Considerable attention has been paid to strongly correlated-electron systems in the study of high- $T_{\mathrm{c}}$ superconductivity since Anderson [1] proposed that its origin may be attributed to magnetic interactions induced by strong Coulomb repulsion. In Anderson's scenario, a Mott insulating phase of an undoped cuprate is assumed to be the resonating-valencebond (RVB) state, composed of electron spin-singlet pairs, and these singlet pairs become charged superconducting pairs when the Mott insulator is doped sufficiently. Although many attempts have been made on lattice models of electrons with interactions, such as the Hubbard model and the $t-J$ model, there is no definite answer to the question of whether this scenario is realized in these concrete microscopic models. These models are usually difficult to analyze theoretically, and exact results are limited except for one dimensional case where Bethe ansatz is available.

One of the exact results in two or more dimensions is obtained by Brandt and Giesekus [2], who constructed the exact ground state of the Hubbard model with infinitely large repulsive on-site interactions on a $d$-dimensional decorated hypercubic lattice. Tasaki [3] generalized the Brandt-Giesekus model to a class of Hubbard models on certain cell structures. He pointed out that the exact ground states in this class of models are regarded as RVB states. He also obtained the singlet pair correlation function on the tree lattice and discussed a possibility of long-range order associated with the singlet pairs and superconductivity. Bares and Lee [4] and Yamanaka et al. [5] treated one dimensional versions of the models and obtained various correlation functions, including the singlet pair correlation function. They found that all the correlation functions decay exponentially with distance and concluded that the system is in an insulating phase. There are no conclusive results about the occurrence (or absence) of superconductivity in the models in two or more dimensions.

For extended Hubbard models including nearest-neighbor interactions in addition to the on-site interactions, there are some exact results relevant to superconductivity. These results are based on the so-called $\eta$-pairing mechanism originally proposed by Yang [6]. By using the $\eta$-pairing mechanism Yang constructed eigenstates of the Hubbard model on the hypercubic lattice and proved that these states, in which all electrons form the $\eta$-pairs, have off-diagonal long-range order and thus are superconducting. Unfortunately, these eigenstates were shown not to be the ground states. But, Essler et al. 7] showed that the $\eta$-pairing states become the exact ground states of an extended Hubbard model with attractive on-site interactions. They also showed that the model with moderately repulsive on-site interactions has ground states in which part of electrons forms the $\eta$-pairs. Possibilities of superconductivity due to the $\eta$-pairing mechanism were discussed in other related models, in particular, in one dimensional systems. See, for example, Refs. [8, [9] and [10] and references therein for more information.

Another rigorous result is a theorem on the Hubbard model with attractive on-site in- 
teractions on bipartite lattices due to S.-Q. Shen and Z.-M. Qiu [11. They proved that these models exhibit off-diagonal long-range order in the ground states. See also Ref. [12] for related results.

At the present time, to draw an exact result about superconductivity in the Hubbard model with on-site repulsion or the $t-J$ model seems to be a formidably difficult task, but we have a chance to obtain one in extended Hubbard models. Although these exactly solvable models have somewhat artificial aspects, they will give us some insight into mechanisms for the phenomenon. In this paper, motivated by this viewpoint, we propose a new exactly solvable model of strongly correlated electrons. It is based on a two dimensional $d$ - $p$ model, a tight binding model of electrons with on-site interactions on the decorated square lattice corresponding to the $\mathrm{CuO}_{2}$ plane. The present new model has the infinitely large repulsive interactions on $\mathrm{Cu}$-sites and furthermore contains correlated-hopping, pair-hopping and attractive charge-charge interactions of electrons. For even numbers of electrons we obtain the exact ground states of the model. A mechanism used to construct the ground states is similar to $\eta$-pairing mechanism, but an electron-pair used here is different from $\eta$-pair. Our electron-pair consists of singlet pairs of electrons on the same O-site, on the nearestneighbor pair of the $\mathrm{Cu}$-sites, and on the nearest-neighbor pair of the $\mathrm{Cu}$ - and $\mathrm{O}$-sites. The constructed exact ground states are expressed as a linear combination of products of these singlet pairs and thus are regarded as RVB states. We also show that these ground states exhibit off-diagonal long-range order, which allow us to construct a ground state with explicit electron-number symmetry breaking.

The recent experimental results of angle-resolved photoemission spectroscopy [13] suggested that electron-phonon coupling is important in high- $T_{\mathrm{c}}$ superconductors and thus a microscopic theory should include this effect. Although this fact does not immediately justify an addition of artificial interactions to a model Hamiltonian, we think that it is important to know what kind of electron-electron interaction, whichever of Coulomb repulsion or electron-phonon coupling (or others) induces this, may stabilize a superconducting state in a theoretical point of view. We hope that our results will be useful in a future study.

In the next section we define the model and state the main result as a theorem. In Section 3, we give some remarks, including the RVB representation and off-diagonal longrange order. In Section 4 we prove the theorem.

\section{Definition of the model and the main result}

We start by describing lattice $\Lambda$ on which our model will be defined. Let $L$ be an arbitrary positive integer and let $D$ be a set of sites

$$
D=\left\{x=\left(x^{(1)}, x^{(2)}\right) \mid x^{(l)} \in \mathbf{Z}, 1 \leq x^{(l)} \leq L \text { for } l=1,2\right\}
$$


with periodic boundary conditions. Let $P$ be a set of sites located at the mid-points of nearest neighbor sites in $D$,

$$
P=\left\{u=x+\delta_{l} / 2 \mid x \in D, l=1,2\right\}
$$

where $\delta_{l}$ is the unit vector along the $l$-axis. Then we define $\Lambda$ as $\Lambda=D \cup P$, which mimics the $\mathrm{CuO}_{2}$ plane. For later use, we also define

$$
P_{x}=\{u|u \in P,| u-x \mid=1 / 2\}
$$

for $x \in D$ and

$$
D_{u}=\{x|x \in D,| x-u \mid=1 / 2\}
$$

for $u \in P$.

Let $c_{i, \sigma}$ and $c_{i, \sigma}^{\dagger}$ be the annihilation and the creation operators, respectively, for an electron with spin $\sigma$ at site $i \in \Lambda$. These operators satisfy the usual anticommutation relations

$$
\left\{c_{i, \sigma}, c_{j, \tau}\right\}=\left\{c_{i, \sigma}^{\dagger}, c_{j, \tau}^{\dagger}\right\}=0
$$

and

$$
\left\{c_{i, \sigma}, c_{j, \tau}^{\dagger}\right\}=\delta_{i j} \delta_{\sigma \tau}
$$

for any $i, j \in \Lambda$ and $\sigma, \tau=\uparrow, \downarrow$. The number operator $n_{i, \sigma}$ is defined as $n_{i, \sigma}=c_{i, \sigma}^{\dagger} c_{i, \sigma}$. We denote by $N_{\mathrm{e}}$ the number of electrons in $\Lambda$ and by $\Phi_{0}$ a state with no electrons.

We assume that repulsive interactions between electrons on $D$-sites are infinitely large and that each of these sites is at most singly occupied. So we consider the Hilbert space $\mathbf{H}_{N_{\mathrm{e}}}$ spanned by the linearly independent states of the form

$$
\Psi\left(A, \boldsymbol{\sigma}_{A} ; B_{\uparrow}, B_{\downarrow}\right)=\left(\prod_{x \in A} c_{x, \sigma_{x}}^{\dagger}\right)\left(\prod_{u \in B_{\uparrow}} c_{u, \uparrow}^{\dagger}\right)\left(\prod_{u \in B_{\downarrow}} c_{u, \downarrow}^{\dagger}\right) \Phi_{0}
$$

with arbitrary subsets $A \subset D, B_{\uparrow}, B_{\downarrow} \subset P$ such that $|A|+\left|B_{\uparrow}\right|+\left|B_{\downarrow}\right|=N_{\mathrm{e}}$. Here $\boldsymbol{\sigma}_{A}$ is a shorthand for a spin configuration $\left(\sigma_{x}\right)_{x \in A}$.

To define the Hamiltonian, we introduce the following new fermion operators:

$$
\begin{gathered}
a_{u, \sigma}=c_{u, \sigma}+\alpha \sum_{x \in D_{u}} c_{x, \sigma} \quad \text { for } u \in P, \\
b_{x, \sigma}=c_{x, \sigma}+\beta \sum_{u \in P_{x}} c_{u, \sigma} \quad \text { for } x \in D, \\
d_{x, \sigma}=\sum_{u \in P_{x}} \mathrm{e}^{-2 \mathrm{i} Q \cdot u} a_{u, \sigma} \quad \text { for } x \in D,
\end{gathered}
$$


where $\alpha$ and $\beta$ are real parameters with $\alpha \neq-\beta$, and $Q=(0, \pi)$. We also introduce operators $n_{u, \sigma}^{a}=a_{u, \sigma}^{\dagger} a_{u, \sigma}, n_{x, \sigma}^{b}=b_{x, \sigma}^{\dagger} b_{x, \sigma}$ and $n_{x, \sigma}^{d}=d_{x, \sigma}^{\dagger} d_{x, \sigma}$. Then, by using these operators, we define

$$
\begin{gathered}
H_{0}=\mathcal{P}_{D}\left(t \sum_{x \in D} \sum_{\sigma=\uparrow, \downarrow} n_{x, \sigma}^{b}+s \sum_{u \in P} \sum_{\sigma=\uparrow, \downarrow} n_{u, \sigma}\right) \mathcal{P}_{D}, \\
H_{\mathrm{int}, 1}=\mathcal{P}_{D}\left(-V_{1} \sum_{x \in D} \sum_{\sigma=\uparrow, \downarrow} n_{x,-\sigma}^{d} n_{x, \sigma}^{b}-W_{1} \sum_{x \in D} \sum_{l=1,2} \sum_{\sigma=\uparrow, \downarrow}\left(n_{x+\delta_{l}, \sigma}+n_{x-\delta_{l}, \sigma}\right) n_{x, \sigma}^{b}\right) \mathcal{P}_{D},
\end{gathered}
$$

and

$$
H_{\mathrm{int}, 2}=\mathcal{P}_{D}\left(-V_{2} \sum_{u \in P} \sum_{\sigma=\uparrow, \downarrow} n_{u,-\sigma}^{a} n_{u, \sigma}-W_{2} \sum_{u \in P} \sum_{x \in D_{u}} \sum_{\sigma=\uparrow, \downarrow} n_{x, \sigma} n_{u, \sigma}\right) \mathcal{P}_{D}
$$

where $t, s, V_{1}, W_{1}, V_{2}$ and $W_{2}$ are real parameters, and $-\sigma$ denotes the spin opposite to $\sigma$. The projection operator $\mathcal{P}_{D}$ which eliminates states with doubly occupied $D$-sites is defined by

$$
\mathcal{P}_{D}=\prod_{x \in D} \mathcal{P}_{x}
$$

with

$$
\mathcal{P}_{x}=\left(1-n_{x, \uparrow} n_{x, \downarrow}\right) .
$$

The Hamiltonian $H_{0}$ is rewritten as

$$
H_{0}=\mathcal{P}_{D}\left(\sum_{i, j \in \Lambda} t_{i j} c_{i, \sigma}^{\dagger} c_{j, \sigma}\right) \mathcal{P}_{D}
$$

with

$$
t_{i j}= \begin{cases}t & \text { if } i=j \in D \\ 2 \beta^{2} t+s & \text { if } i=j \in P \\ \beta t & \text { if }|i-j|=\frac{1}{2} \\ \beta^{2} t & \text { if } i \neq j, i, j \in P_{x} \text { for some } x \in D \\ 0 & \text { otherwise, }\end{cases}
$$

(see Fig. (1) and it describes quantum mechanical motion of electrons feeling infinitely large repulsive interactions at $D$-sites. The Hamiltonians $H_{\text {int, } 1}$ and $H_{\text {int, } 2}$ correspond to correlatedhopping, pair-hopping, charge-charge, and spin-spin interactions. We can observe this by rewriting, for example, $H_{\mathrm{int}, 2}$ as

$$
H_{\mathrm{int}, 2}=\mathcal{P}_{D}\left(\sum _ { u \in P } \left(-\alpha^{2} V_{2} \sum_{x, y \in D_{u} ; x \neq y} \sum_{\sigma=\uparrow, \downarrow} c_{x,-\sigma}^{\dagger} c_{y,-\sigma} n_{u, \sigma}\right.\right.
$$




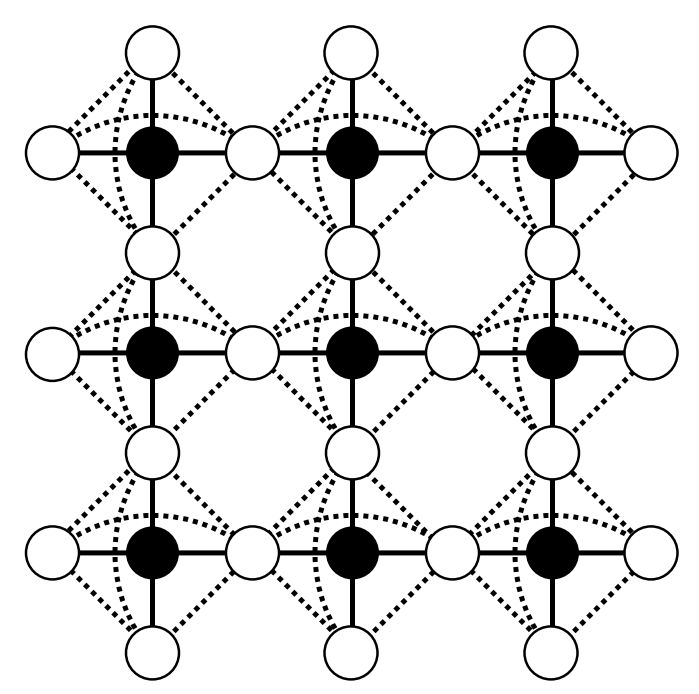

Figure 1: The lattice structure and the hopping matrix elements of $H_{0}$. The filled circles and the open circles correspond to $D$-sites and $P$-sites, respectively. The on-site potential of $D$-sites is $t$ and that of $P$-sites is $2 \beta^{2} t+s$. The solid lines and the dashed lines represent the hopping matrix elements, $\beta t$ and $\beta^{2} t$, respectively.

$$
\begin{aligned}
& -\alpha V_{2} \sum_{x \in D_{u}} \sum_{\sigma=\uparrow, \downarrow}\left(c_{x,-\sigma}^{\dagger} c_{u,-\sigma}+c_{u,-\sigma}^{\dagger} c_{x,-\sigma}\right) n_{u, \sigma}-2 V_{2} n_{u, \uparrow} n_{u, \downarrow} \\
& \left.\left.-\frac{1}{2}\left(\alpha^{2} V_{2}+W_{2}\right) \sum_{x \in D_{u}} n_{x} n_{u}+2\left(\alpha^{2} V_{2}-W_{2}\right) \sum_{x \in D_{u}} S_{x}^{(3)} S_{u}^{(3)}\right)\right) \mathcal{P}_{D},
\end{aligned}
$$

where $n_{i}=n_{i, \uparrow}+n_{i \downarrow}$ and $S_{i}^{(3)}=\left(n_{i, \uparrow}-n_{i \downarrow}\right) / 2$. The Hamiltonian $H_{\text {int }, 1}$ can be rewritten as well, but that has a somewhat complicated form. We note that the interactions $H_{\text {int, } 1}$ and $H_{\text {int,2 }}$ conserve the electron number and the eigenvalue of $\sum_{i \in \Lambda} S_{i}^{(3)}$, but they do not possess the spin $\mathrm{SU}(2)$ symmetry.

In this paper we consider the Hamiltonian

$$
H=H_{0}+H_{\mathrm{int}, 1}+H_{\mathrm{int}, 2}
$$

with the parameters satisfying $t=4\left(1+\alpha^{2}\right) V_{1}, W_{1}=\alpha^{2} V_{1} s=\left(1+2 \alpha^{2}\right) V_{2}, W_{2}=\alpha^{2} V_{2}$ and $V_{1}, V_{2}>0$. In this case, the direct spin-spin interaction terms vanish and the charge-charge interactions are attractive.

In our construction of exact ground states, it is crucial to rewrite $H$ as

$$
H=\sum_{x \in D} H_{x}+\sum_{u \in P} H_{u}
$$


with

$$
H_{x}=\mathcal{P}_{D}\left(V_{1} \sum_{\sigma=\uparrow, \downarrow} b_{x, \sigma}^{\dagger} d_{x,-\sigma} \mathcal{P}_{D} d_{x,-\sigma}^{\dagger} b_{x, \sigma}\right) \mathcal{P}_{D}
$$

for $x \in D$ and

$$
H_{u}=\mathcal{P}_{D}\left(V_{2} \sum_{\sigma=\uparrow, \downarrow} c_{u, \sigma}^{\dagger} a_{u,-\sigma} \mathcal{P}_{D} a_{u,-\sigma}^{\dagger} c_{u, \sigma}\right) \mathcal{P}_{D}
$$

for $u \in P$. Here we used the operator identities [2, 14]

$$
\mathcal{P}_{D} c_{i, \sigma} \mathcal{P}_{D} c_{j, \sigma}^{\dagger} \mathcal{P}_{D}= \begin{cases}-\mathcal{P}_{D} c_{j, \sigma}^{\dagger} c_{i, \sigma} \mathcal{P}_{D} & \text { if } i \neq j \\ \mathcal{P}_{D}\left(1-n_{i, \uparrow}-n_{i, \downarrow}\right) \mathcal{P}_{D} & \text { if } i=j \in D \\ \mathcal{P}_{D}\left(1-n_{i, \sigma}\right) \mathcal{P}_{D} & \text { if } i=j \in P\end{cases}
$$

and

$$
\begin{aligned}
& \mathcal{P}_{x} c_{i, \sigma} \mathcal{P}_{x}=c_{i, \sigma} \mathcal{P}_{x}, \\
& \mathcal{P}_{x} c_{i, \sigma}^{\dagger} \mathcal{P}_{x}=\mathcal{P}_{x} c_{i, \sigma}^{\dagger} .
\end{aligned}
$$

Operator identities (2.24) and (2.25) follow from straightforward calculations, and (2.23) is derived by using $n_{x, \uparrow} n_{x, \downarrow} \mathcal{P}_{x}=0$ and

$$
\begin{aligned}
& c_{i, \sigma} \mathcal{P}_{x}= \begin{cases}\left(1-n_{x,-\sigma}\right) c_{x, \sigma}=\mathcal{P}_{x}\left(1-n_{x,-\sigma}\right) c_{x, \sigma} & \text { if } i=x ; \\
\mathcal{P}_{x} c_{i, \sigma} & \text { otherwise, }\end{cases} \\
& \mathcal{P}_{x} c_{i, \sigma}^{\dagger}= \begin{cases}c_{x, \sigma}^{\dagger}\left(1-n_{x,-\sigma}\right)=c_{x, \sigma}^{\dagger}\left(1-n_{x,-\sigma}\right) \mathcal{P}_{x} & \text { if } i=x ; \\
c_{i, \sigma}^{\dagger} \mathcal{P}_{x} & \text { otherwise, }\end{cases}
\end{aligned}
$$

which also follow from simple calculations. We note that $H_{i}$ with $i \in \Lambda$ are positive semidefinite operators and thus a zero-energy state for all $H_{i}$ (if it exists) is a ground state.

Let us define

$$
\zeta^{\dagger}=\sum_{u \in P} \mathrm{e}^{2 \mathrm{i} Q \cdot u} a_{u, \uparrow}^{\dagger} a_{u, \downarrow}^{\dagger}
$$

which corresponds to a singlet two-electron state. The main result in this paper is the following theorem:

Theorem 2.1 Fix the electron number $N_{\mathrm{e}}$ and suppose that $N_{\mathrm{e}} \leq 2|P|$. When $N_{\mathrm{e}}$ is even, the ground state $\Phi_{\mathrm{G}, N_{\mathrm{e}}}$ of $H$ is unique and is given by

$$
\Phi_{\mathrm{G}, N_{\mathrm{e}}}=\mathcal{P}_{D}\left(\zeta^{\dagger}\right)^{\frac{N_{\mathrm{e}}}{2}} \Phi_{0}=\left(\mathcal{P}_{D} \zeta^{\dagger}\right)^{\frac{N_{\mathrm{e}}}{2}} \Phi_{0}
$$

which satisfies $H \Phi_{\mathrm{G}, N_{\mathrm{e}}}=0$. For odd $N_{\mathrm{e}}$, the ground state energy is positive.

It is noted that the ground state energy for odd $N_{\mathrm{e}}$ may converge to zero as $L \rightarrow \infty$. Whether this is the case or not should be clarified in a future study. 


\section{Some Remarks}

It is easy to verify the relation

$$
\zeta^{\dagger}=\sum_{x \in D} \sum_{l=1,2} \mathrm{e}^{\mathrm{i} Q \cdot \delta_{l}}\left(\alpha^{2} f_{x, x+\delta_{l}}^{\dagger}+\alpha f_{x, x+\delta_{l} / 2}^{\dagger}+\alpha f_{x, x-\delta_{l} / 2}^{\dagger}\right)+\sum_{u \in P} \mathrm{e}^{2 \mathrm{i} Q \cdot u} c_{u, \uparrow}^{\dagger} c_{u, \downarrow}^{\dagger}
$$

where $f_{i, j}^{\dagger}=c_{i, \uparrow}^{\dagger} c_{j, \downarrow}^{\dagger}+c_{j, \uparrow}^{\dagger} c_{i, \downarrow}^{\dagger}$ is the creation operator for the valence-bond (singlet pair) on sites $i$ and $j$. Furthermore the projection operator $\mathcal{P}_{D}$ eliminates the terms of the form

$$
f_{x, i}^{\dagger} f_{x, j}^{\dagger} \cdots \Phi_{0}=-c_{x, \uparrow}^{\dagger} c_{x, \downarrow}^{\dagger} f_{i, j}^{\dagger} \cdots \Phi_{0}
$$

when we express $\Phi_{\mathrm{G}, N_{\mathrm{e}}}$ by using $f_{i, j}^{\dagger}$ and $c_{u, \uparrow}^{\dagger} c_{u, \downarrow}^{\dagger}$. Thus the ground state $\Phi_{\mathrm{G}, N_{\mathrm{e}}}$ has the form of the RVB state which is a linear combination of products of on-site singlet pairs $c_{u, \uparrow}^{\dagger} c_{u, \downarrow}^{\dagger}$ in $P$ and the valence-bonds $f_{x, x+\delta_{l}}^{\dagger}$ and $f_{x, x \pm \delta_{l} / 2}^{\dagger}$. It is noted that the weight for the singlet pairs $f_{x, x+\delta_{l}}^{\dagger}$ on the nearest-neighbor $D$-sites becomes relatively large for $1 / \alpha \ll 1$.

The term $-V_{1} n_{x,-\sigma}^{d} n_{x, \sigma}^{b}$ in interaction Hamiltonian $H_{\mathrm{int}, 1}$ is rewritten as

$$
-V_{1} n_{x,-\sigma}^{d} n_{x, \sigma}^{b}=-4\left(1+\alpha^{2}\right)\left(1+4 \beta^{2}\right) V_{1}+4\left(1+\alpha^{2}\right) V_{1} b_{x, \sigma} b_{x, \sigma}^{\dagger}+V_{1} d_{x,-\sigma} b_{x, \sigma}^{\dagger} b_{x, \sigma} d_{x,-\sigma}^{\dagger} .
$$

From this expression, we find that $-V_{1} n_{x,-\sigma}^{d} n_{x, \sigma}^{b}$ is bounded below by $-4\left(1+\alpha^{2}\right)\left(1+4 \beta^{2}\right) V_{1}$ and furthermore that it is attained by states of the form $b_{x, \sigma}^{\dagger} d_{x,-\sigma}^{\dagger} \cdots \Phi_{0}$. Therefore this interaction term is describing an attractive and magnetic interaction between localized singleelectron states corresponding to fermion operators $b_{x, \sigma}$ and $d_{x, \sigma}$. The other terms can be rewritten into similar forms and interpreted as attractive and magnetic interactions as well. Our model says that attractive magnetic interactions between localized states together with the strong on-site repulsion can stabilize the condensed RVB states consisting of local singlet pairs.

The explicit expression of the ground states makes it possible to evaluate order parameters. We first note that, in the case of $\alpha=0, \Phi_{\mathrm{G}, N_{\mathrm{e}}}$ is reduced to the $\eta$-pairing state on the $P$-sites, and therefore the ground states have off-diagonal long-range order.

For $\alpha \neq 0$ we can prove the existence of off-diagonal long-range order as follows. Let us introduce order parameters

$$
\Delta_{1, \Lambda}=\mathcal{P}_{D}\left(\frac{1}{|P|} \sum_{u \in P} \mathrm{e}^{-2 \mathrm{i} Q \cdot u} c_{u, \downarrow} c_{u, \uparrow}\right) \mathcal{P}_{D}
$$

and

$$
\Delta_{2, \Lambda}=\mathcal{P}_{D}\left(\frac{1}{|P|} \sum_{u \in P} \mathrm{e}^{-2 \mathrm{i} Q \cdot u} a_{u, \downarrow} a_{u, \uparrow}\right) \mathcal{P}_{D}=\frac{1}{|P|} \mathcal{P}_{D} \zeta \mathcal{P}_{D}
$$


Noting the anticommutation relation

$$
\left\{c_{u, \sigma}, a_{u^{\prime}, \sigma}^{\dagger}\right\}=\delta_{u u^{\prime}}
$$

for $u, u^{\prime} \in P$, one finds the commutation relation

$$
\left[\Delta_{1, \Lambda}, \mathcal{P}_{D} \zeta^{\dagger} \mathcal{P}_{D}\right]=\mathcal{P}_{D}\left(\frac{1}{|P|} \sum_{u \in P}\left(1-a_{u, \uparrow}^{\dagger} c_{u, \uparrow}-a_{u, \downarrow}^{\dagger} c_{u, \downarrow}\right)\right) \mathcal{P}_{D}
$$

and

$$
\mathcal{P}_{D} \sum_{u \in P}\left(1-a_{u, \uparrow}^{\dagger} c_{u, \uparrow}-a_{u, \downarrow}^{\dagger} c_{u, \downarrow}\right) \mathcal{P}_{D} \Phi_{\mathrm{G}, N_{\mathrm{e}}}=\left(|P|-N_{\mathrm{e}}\right) \Phi_{\mathrm{G}, N_{\mathrm{e}}} .
$$

These two relations lead to

$$
\Delta_{1, \Lambda} \Phi_{\mathrm{G}, N_{\mathrm{e}}}=\left(\frac{N_{\mathrm{e}}}{2}-\frac{1}{|P|} \frac{N_{\mathrm{e}}}{2}\left(\frac{N_{\mathrm{e}}}{2}-1\right)\right) \Phi_{\mathrm{G}, N_{\mathrm{e}}-2} .
$$

Therefore we obtain

$$
\left\langle\Delta_{2, \Lambda}^{\dagger} \Delta_{1, \Lambda}\right\rangle_{\Lambda, N_{\mathrm{e}}}=\frac{1}{|P|}\left(\frac{N_{\mathrm{e}}}{2}-\frac{1}{|P|} \frac{N_{\mathrm{e}}}{2}\left(\frac{N_{\mathrm{e}}}{2}-1\right)\right),
$$

where the expectation value $\langle\cdots\rangle_{\Lambda, N_{e}}$ is defined by

$$
\langle\cdots\rangle_{\Lambda, N_{\mathrm{e}}}=\frac{\left(\Phi_{\mathrm{G}, N_{\mathrm{e}}}, \cdots \Phi_{\mathrm{G}, N_{\mathrm{e}}}\right)}{\left(\Phi_{\mathrm{G}, N_{\mathrm{e}}}, \Phi_{\mathrm{G}, N_{\mathrm{e}}}\right)} .
$$

We write $\mu_{\Lambda, N_{\mathrm{e}}}$ for the right-hand-side of (3.10). Then, by using the Schwarz inequality

$$
\left|\left\langle\Delta_{2, \Lambda}^{\dagger} \Delta_{1, \Lambda}\right\rangle_{\Lambda, N_{\mathrm{e}}}\right|^{2} \leq\left\langle\Delta_{2, \Lambda}^{\dagger} \Delta_{2, \Lambda}\right\rangle_{\Lambda, N_{\mathrm{e}}}\left\langle\Delta_{1, \Lambda}^{\dagger} \Delta_{1, \Lambda}\right\rangle_{\Lambda, N_{\mathrm{e}}},
$$

and inequalities $\left\langle\Delta_{1, \Lambda}^{\dagger} \Delta_{1, \Lambda}\right\rangle_{\Lambda, N_{\mathrm{e}}} \leq 1$ and $\left\langle\Delta_{2, \Lambda}^{\dagger} \Delta_{2, \Lambda}\right\rangle_{\Lambda, N_{\mathrm{e}}} \leq(1+2 \alpha)^{2}$, we find

$$
\frac{\mu_{\Lambda, N_{\mathrm{e}}}^{2}}{\left(1+2 \alpha^{2}\right)^{2}} \leq\left\langle\Delta_{1, \Lambda}^{\dagger} \Delta_{1, \Lambda}\right\rangle_{\Lambda, N_{\mathrm{e}}} \leq 1
$$

and

$$
\mu_{\Lambda, N_{\mathrm{e}}}^{2} \leq\left\langle\Delta_{2, \Lambda}^{\dagger} \Delta_{2, \Lambda}\right\rangle_{\Lambda, N_{\mathrm{e}}} \leq\left(1+2 \alpha^{2}\right)^{2} .
$$

Let $\left\{\Phi_{\mathrm{G}, L}^{(\nu)}\right\}_{L=0}^{\infty}$ be a sequence of ground states $\Phi_{\mathrm{G}, N_{\mathrm{e}}}$ on the lattice with side-length $L$ such that the electron filling $N_{\mathrm{e}} /(2|\Lambda|)=N_{\mathrm{e}} /\left(6 L^{2}\right)$ converges to $\nu$ as $L \rightarrow \infty$, and let $\left\{\langle\cdots\rangle_{L}^{(\nu)}\right\}_{L=0}^{\infty}$ 
be a sequence of expectation values with respect to $\Phi_{\mathrm{G}, L}^{(\nu)}$. Then from inequalities (3.13) and (3.14) we have

$$
\liminf _{L \rightarrow \infty}\left\langle\Delta_{1, \Lambda}^{\dagger} \Delta_{1, \Lambda}\right\rangle_{L}^{(\nu)} \geq \frac{\mu_{\nu}^{2}}{\left(1+2 \alpha^{2}\right)^{2}}
$$

and

$$
\liminf _{L \rightarrow \infty}\left\langle\Delta_{2, \Lambda}^{\dagger} \Delta_{2, \Lambda}\right\rangle_{L}^{(\nu)} \geq \mu_{\nu}^{2}
$$

where $\mu_{\nu}=\frac{3 \nu}{2}\left(1-\frac{3 \nu}{2}\right)$, which imply the existence of off-diagonal long-range order for $0<$ $\nu<2 / 3$.

Now we can construct a ground state with explicit electron-number symmetry breaking [15]. Let us define

$$
\Phi_{\mathrm{G}, N_{\mathrm{e}}}^{\prime}=\Phi_{\mathrm{G}, N_{\mathrm{e}}}+\frac{\Delta_{2, \Lambda}^{\dagger}}{\sqrt{\left\langle\Delta_{2, \Lambda} \Delta_{2, \Lambda}^{\dagger}\right\rangle_{\Lambda, N_{\mathrm{e}}}}} \Phi_{\mathrm{G}, N_{\mathrm{e}}}
$$

which is also a zero-energy state. We note that the limit infimum of $\left\langle\Delta_{2, \Lambda} \Delta_{2, \Lambda}^{\dagger}\right\rangle_{L}^{(\nu)}$ is bounded below by $\mu_{\nu}^{2}$, since

$$
\left\langle\Delta_{1, \Lambda} \Delta_{2, \Lambda}^{\dagger}\right\rangle_{\Lambda, N_{\mathrm{e}}}=\left\langle\Delta_{2, \Lambda}^{\dagger} \Delta_{1, \Lambda}\right\rangle_{\Lambda, N_{\mathrm{e}}}+\frac{1}{|P|^{2}}\left(|P|-N_{\mathrm{e}}\right)
$$

which follows from (3.7). Since two states with different electron number are orthogonal, we find that

$$
\left\langle\Delta_{2, \Lambda}\right\rangle_{\Lambda, N_{\mathrm{e}}}^{\prime}=\frac{\left(\Phi_{\mathrm{G}, N_{\mathrm{e}}}^{\prime}, \Delta_{2, \Lambda} \Phi_{\mathrm{G}, N_{\mathrm{e}}}^{\prime}\right)}{\left(\Phi_{\mathrm{G}, N_{\mathrm{e}}}^{\prime}, \Phi_{\mathrm{G}, N_{\mathrm{e}}}^{\prime}\right)}=\frac{1}{2} \sqrt{\left\langle\Delta_{2, \Lambda} \Delta_{2, \Lambda}^{\dagger}\right\rangle_{\Lambda, N_{\mathrm{e}}}}
$$

Therefore, the limit infimum of the sequence of $\left\langle\Delta_{2, \Lambda}\right\rangle_{\Lambda, N_{\mathrm{e}}}^{\prime}$ obtained by using $\left\{\Phi_{\mathrm{G}, L}^{(\nu)}\right\}_{L=0}^{\infty}$ with (3.17) is bounded below by $\mu_{\nu} / 2$, which implies electron-number symmetry breaking.

We finally remark about extensions of the present model. It is possible to construct similar models in one, three and more dimensions by the same method. The model in three or more dimensions may exhibit a finite temperature phase transition. It is also possible to construct models whose ground states are written as $\left(\zeta^{\dagger}\right)^{\frac{N_{\mathrm{e}}}{2}} \Phi_{0}$. The details will appear elsewhere.

\section{Proof}

Proof of Theorem [2.1. We first prove that $\Phi_{\mathrm{G}, N_{\mathrm{e}}}$ in (2.29) is a zero-energy state for all $H_{i}$ and thus is a ground state. Using the anticommutation relations (3.6) and

$$
\left\{c_{x, \sigma}, a_{u, \sigma}^{\dagger}\right\}=\alpha \chi\left[u \in P_{x}\right]
$$


for $x \in D$, where $\chi$ ["event"] takes 1 if "event" is true and takes 0 otherwise, one finds the following two commutation relations:

$$
\left[c_{u, \sigma}, \zeta^{\dagger}\right]=\sigma \mathrm{e}^{2 \mathrm{i} Q \cdot u} a_{u,-\sigma}^{\dagger}
$$

for $u \in P$ and

$$
\left[c_{x, \sigma}, \zeta^{\dagger}\right]=\sigma \alpha \sum_{u \in P_{x}} \mathrm{e}^{2 \mathrm{i} Q \cdot u} a_{u,-\sigma}^{\dagger}
$$

for $x \in D$. (In the right-hand-sides of (4.2) and (4.3), the coefficients $\sigma=\uparrow$ and $\downarrow$ are regarded as +1 and -1 , respectively. We will use this convention in the following.) By using operator identities (2.25), (2.26) and commutation relation (4.2), we find

$$
\begin{aligned}
\left(\mathcal{P}_{D} a_{u,-\sigma}^{\dagger} c_{u, \sigma}\right) \mathcal{P}_{D} \zeta^{\dagger} & =\mathcal{P}_{D} a_{u,-\sigma}^{\dagger} c_{u, \sigma} \zeta^{\dagger} \\
& =\mathcal{P}_{D} a_{u,-\sigma}^{\dagger}\left(\zeta^{\dagger} c_{u, \sigma}+\sigma \mathrm{e}^{2 \mathrm{i} Q \cdot u} a_{u,-\sigma}^{\dagger}\right) \\
& =\mathcal{P}_{D} \zeta^{\dagger}\left(\mathcal{P}_{D} a_{u,-\sigma}^{\dagger} c_{u, \sigma}\right) .
\end{aligned}
$$

To get the third line, we used $\left(a_{u,-\sigma}^{\dagger}\right)^{2}=0$. This implies that $\left(\mathcal{P}_{D} a_{u,-\sigma}^{\dagger} c_{u, \sigma}\right)\left(\mathcal{P}_{D} \zeta^{\dagger}\right)^{\frac{N_{\mathrm{e}}}{2}} \Phi_{0}=0$, and thus $H_{u} \Phi_{\mathrm{G}, N_{\mathrm{e}}}=0$ for all $u \in P$. Similarly, by using (2.25), (2.26) and commutation relations (4.2), (4.3) we have

$$
\begin{aligned}
\left(\mathcal{P}_{D} d_{x,-\sigma}^{\dagger} \sum_{u \in P_{x}} c_{u, \sigma}\right) \mathcal{P}_{D} \zeta^{\dagger} & =\left(\mathcal{P}_{D} d_{x,-\sigma}^{\dagger} \sum_{u \in P_{x}} c_{u, \sigma}\right) \zeta^{\dagger} \\
& =\mathcal{P}_{D} d_{x,-\sigma}^{\dagger} \zeta^{\dagger} \sum_{u \in P_{x}} c_{u, \sigma}+\sigma \mathcal{P}_{D} d_{x,-\sigma}^{\dagger} \sum_{u \in P_{x}} \mathrm{e}^{2 \mathrm{i} Q \cdot u} a_{u,-\sigma}^{\dagger} \\
& =\mathcal{P}_{D} \zeta^{\dagger}\left(\mathcal{P}_{D} d_{x,-\sigma}^{\dagger} \sum_{u \in P_{x}} c_{u, \sigma}\right)
\end{aligned}
$$

and

$$
\begin{aligned}
\left(\mathcal{P}_{D} d_{x,-\sigma}^{\dagger} c_{x, \sigma}\right) \mathcal{P}_{D} \zeta^{\dagger}= & \mathcal{P}_{D}\left(1-n_{x,-\sigma}\right) d_{x,-\sigma}^{\dagger} c_{x, \sigma} \zeta^{\dagger} \\
= & \mathcal{P}_{D}\left(1-n_{x,-\sigma}\right) d_{x,-\sigma}^{\dagger} \zeta^{\dagger} c_{x, \sigma} \\
& +\sigma \alpha \mathcal{P}_{D}\left(1-n_{x,-\sigma}\right) d_{x,-\sigma}^{\dagger} \sum_{u \in P_{x}} \mathrm{e}^{2 \mathrm{i} Q \cdot u} a_{u,-\sigma}^{\dagger} \\
= & \mathcal{P}_{D}\left(1-n_{x,-\sigma}\right) \zeta^{\dagger}\left(\mathcal{P}_{D} d_{x,-\sigma}^{\dagger} c_{x, \sigma}\right) .
\end{aligned}
$$

Here we also used $\left\{c_{x,-\sigma}, d_{x,-\sigma}^{\dagger}\right\}=0$ and $\left(d_{x,-\sigma}^{\dagger}\right)^{2}=0$. It follows from (4.5) and (4.6) that $\left(\mathcal{P}_{D} d_{x,-\sigma}^{\dagger} b_{x, \sigma}\right)\left(\mathcal{P}_{D} \zeta^{\dagger}\right)^{\frac{N_{\mathrm{e}}}{2}} \Phi_{0}=0$, i.e., $H_{x} \Phi_{\mathrm{G}, N_{\mathrm{e}}}=0$ for all $x \in D$. Therefore we conclude that $H \Phi_{\mathrm{G}, N_{\mathrm{e}}}=0$.

The proof for the other statements in the theorem relies on the following lemma, which will be proved later. 
Lemma 4.1 Any zero-energy state $\Phi$ for $H$ in the Hilbert space $\bigoplus_{N_{\mathrm{e}}=0}^{2|P|} \mathbf{H}_{N_{\mathrm{e}}}$ is written as

$$
\Phi=\sum_{B \subset P} \phi(B) \mathcal{P}_{D}\left(\prod_{u \in B} a_{u, \uparrow}^{\dagger}\right)\left(\prod_{u \in B} a_{u, \downarrow}^{\dagger}\right) \Phi_{0},
$$

where $\phi(B)$ are real coefficients. Furthermore coefficients satisfy $|\phi(B)|=\left|\phi\left(B^{\prime}\right)\right|$ for any $B, B^{\prime} \subset P$ such that $|B|=\left|B^{\prime}\right|$.

It immediately follows from Lemma 4.1 that the ground state energy for odd $N_{\mathrm{e}}$ is always positive. The remaining task is to prove the uniqueness. Suppose that there are two zeroenergy states for fixed even $N_{\mathrm{e}}$. Then, an arbitrary linear combination of these states is also a zero-energy state, which should satisfy Lemma 4.1. However, we can make a suitable linear combination so that a coefficient $\phi\left(B_{0}\right)$ for a subset $B_{0}$ will be vanishing, and this leads to the conclusion that all the other coefficients are also vanishing. This is contradicting with the assumption, and therefore the ground state is unique. This completes the proof of Theorem 2.1]

Before proceeding to the proof of Lemma 4.1, we prove the following lemma.

Lemma 4.2 For $A \subset D$, spin configuration $\boldsymbol{\sigma}_{A}$ and $B_{\uparrow}, B_{\downarrow} \subset P$, define

$$
\Phi\left(A, \boldsymbol{\sigma}_{A} ; B_{\uparrow}, B_{\downarrow}\right)=\left(\prod_{x \in A} c_{x, \sigma_{x}}^{\dagger}\right)\left(\prod_{u \in B_{\uparrow}} a_{u, \uparrow}^{\dagger}\right)\left(\prod_{u \in B_{\downarrow}} a_{u, \downarrow}^{\dagger}\right) \Phi_{0} .
$$

Then, the states $\mathcal{P}_{D} \Phi\left(A, \boldsymbol{\sigma}_{A} ; B_{\uparrow}, B_{\downarrow}\right)$ are linearly independent, and the collection of these states with $|A|+\left|B_{\uparrow}\right|+\left|B_{\downarrow}\right|=N_{\mathrm{e}}$ spans the Hilbert space $\mathbf{H}_{N_{\mathrm{e}}}$.

Proof of Lemma 4.2. Consider a linear combination

$$
\Phi=\sum_{A \subset D} \sum_{\boldsymbol{\sigma}_{A}} \sum_{B_{\uparrow}, B_{\downarrow} \subset P} \phi\left(A, \boldsymbol{\sigma}_{A} ; B_{\uparrow}, B_{\downarrow}\right) \mathcal{P}_{D} \Phi\left(A, \boldsymbol{\sigma}_{A} ; B_{\uparrow}, B_{\downarrow}\right),
$$

where $\sum \boldsymbol{\sigma}_{A}$ means the sum taken over all spin configurations. We suppose that $\Phi=0$. From the anticommutation relation (3.6) it follows that the inner product

$$
\left(\Psi\left(A^{\prime}, \boldsymbol{\sigma}_{A^{\prime}}^{\prime} ; B_{\uparrow}^{\prime}, B_{\downarrow}^{\prime}\right), \mathcal{P}_{D} \Phi\left(A, \boldsymbol{\sigma}_{A} ; B_{\uparrow}, B_{\downarrow}\right)\right)
$$

is zero if both $B_{\uparrow}^{\prime} \subset B_{\uparrow}$ and $B_{\downarrow}^{\prime} \subset B_{\downarrow}$ do not hold. (See (2.7) for the definition of $\Psi\left(A, \boldsymbol{\sigma}_{A} ; B_{\uparrow}, B_{\downarrow}\right)$.) We furthermore have that

$$
\left(\Psi\left(A^{\prime}, \boldsymbol{\sigma}_{A^{\prime}}^{\prime} ; B_{\uparrow}, B_{\downarrow}\right), \mathcal{P}_{D} \Phi\left(A, \boldsymbol{\sigma}_{A} ; B_{\uparrow}, B_{\downarrow}\right)\right)=0
$$

for $A^{\prime} \neq A$ and that

$$
\left(\Psi\left(A, \boldsymbol{\sigma}_{A}^{\prime} ; B_{\uparrow}, B_{\downarrow}\right), \mathcal{P}_{D} \Phi\left(A, \boldsymbol{\sigma}_{A} ; B_{\uparrow}, B_{\downarrow}\right)\right)=\chi\left[\sigma_{x}^{\prime}=\sigma_{x} \text { for all } x \in A\right] .
$$


Thus we obtain from $\left(\Psi\left(A, \boldsymbol{\sigma}_{A} ; P, P\right), \Phi\right)=0$ that $\phi\left(A, \boldsymbol{\sigma}_{A} ; P, P\right)=0$ for any $A \subset D$ and spin configuration $\boldsymbol{\sigma}_{A}$. Then, examining

$$
\left(\Psi\left(A, \boldsymbol{\sigma}_{A} ; P \backslash\{u\}, P\right), \Phi\right)=0
$$

for $u \in P$, we find that $\phi\left(A, \boldsymbol{\sigma}_{A} ; P \backslash\{u\}, P\right)=0$. Similarly, examining

$$
\left(\Psi\left(A, \boldsymbol{\sigma}_{A} ; P \backslash \bar{B}_{\uparrow}, P \backslash \bar{B}_{\downarrow}\right), \Phi\right)=0
$$

for $\bar{B}_{\uparrow}, \bar{B}_{\downarrow} \subset P$ with $\left|\bar{B}_{\uparrow}\right|,\left|\bar{B}_{\downarrow}\right|=0,1,2, \ldots|P|$ repeatedly, we conclude that all the coefficients $\phi\left(A, \boldsymbol{\sigma}_{A} ; B_{\uparrow}, B_{\downarrow}\right)$ are vanishing. This proves the first claim, and the second claim is now trivial.

Proof of Lemma 4.1. Suppose that $\Phi$ is an arbitrary zero-energy state for $H$ in $\bigoplus_{N_{\mathrm{e}}=0}^{2|P|} \mathbf{H}_{N_{\mathrm{e}}}$. By using the basis states $\mathcal{P}_{D} \Phi\left(A, \boldsymbol{\sigma}_{A} ; B_{\uparrow}, B_{\downarrow}\right)$ with $|A|+\left|B_{\uparrow}\right|+\left|B_{\downarrow}\right| \leq 2|P|$, we represent $\Phi$ as

$$
\Phi=\sum_{A \subset D} \sum_{\boldsymbol{\sigma}_{A}} \sum_{B_{\uparrow}, B_{\downarrow} \subset P} \phi\left(A, \boldsymbol{\sigma}_{A} ; B_{\uparrow}, B_{\downarrow}\right) \mathcal{P}_{D} \Phi\left(A, \boldsymbol{\sigma}_{A} ; B_{\uparrow}, B_{\downarrow}\right) .
$$

The sum is restricted to $|A|+\left|B_{\uparrow}\right|+\left|B_{\downarrow}\right| \leq 2|P|$, but, here and in the following, we do not written down this restriction for notational simplicity.

Since the local Hamiltonian $H_{i}$ is the sum of two positive semidefinite operators, zeroenergy state $\Phi$ for $H$ must be annihilated by these operators for any $i \in \Lambda$.

We first examine the case $i=u \in P$. In this case the condition $H_{u} \Phi=0$ is equivalent to

$$
\mathcal{P}_{D} a_{u,-\sigma}^{\dagger} c_{u, \sigma} \mathcal{P}_{D} \Phi=0
$$

for $\sigma=\uparrow, \downarrow$. For $\sigma=\uparrow$ the left hand side of (4.16) becomes

$$
\begin{aligned}
\sum_{A \subset D} \sum_{\boldsymbol{\sigma}_{A}} \sum_{B_{\uparrow}, B_{\downarrow} \subset P} \operatorname{sgn}\left[u ; B_{\uparrow}, B_{\downarrow}\right] \chi\left[u \in B_{\uparrow}\right] \chi\left[u \notin B_{\downarrow}\right] \\
\times \phi\left(A, \boldsymbol{\sigma}_{A} ; B_{\uparrow}, B_{\downarrow}\right) \mathcal{P}_{D} \Phi\left(A, \boldsymbol{\sigma}_{A} ; B_{\uparrow} \backslash\{u\}, B_{\downarrow} \cup\{u\}\right),
\end{aligned}
$$

where $\operatorname{sgn}\left[u ; B_{\uparrow}, B_{\downarrow}\right]$ is a sign factor arising from exchanges of fermion operators. Since all the terms in (4.17) are linearly independent, we have the condition $\phi\left(A, \boldsymbol{\sigma}_{A} ; B_{\uparrow}, B_{\downarrow}\right)=0$ for $B_{\uparrow}, B_{\downarrow}$ such that $u \in B_{\uparrow}$ and $u \notin B_{\downarrow}$. A similar calculation for $\sigma=\downarrow$ yields $\phi\left(A, \boldsymbol{\sigma}_{A} ; B_{\uparrow}, B_{\downarrow}\right)=$ 0 for $B_{\uparrow}, B_{\downarrow}$ such that $u \notin B_{\uparrow}$ and $u \in B_{\downarrow}$. Since these conditions must be satisfied for all $u \in P$, we obtain $\phi\left(A, \boldsymbol{\sigma}_{A} ; B_{\uparrow}, B_{\downarrow}\right)=0$ for $B_{\uparrow} \neq B_{\downarrow}$.

So far we have shown that a zero-energy state $\Phi$ can be expanded as

$$
\Phi=\sum_{A \subset D} \sum_{\boldsymbol{\sigma}_{A}} \sum_{B \subset P} \phi\left(A, \boldsymbol{\sigma}_{A} ; B\right) \mathcal{P}_{D} \Phi\left(A, \boldsymbol{\sigma}_{A} ; B, B\right)
$$


where $\phi\left(A, \boldsymbol{\sigma}_{A} ; B\right)=\phi\left(A, \boldsymbol{\sigma}_{A} ; B, B\right)$. We further derive conditions on $\phi\left(A, \boldsymbol{\sigma}_{A} ; B\right)$ from $H_{i} \Phi=0$ with $i=x \in D$, which is equivalent to

$$
\mathcal{P}_{D} d_{x,-\sigma}^{\dagger} b_{x, \sigma} \mathcal{P}_{D} \Phi=0
$$

for $\sigma=\uparrow, \downarrow$. The left-hand-side of (4.19) can be expanded by using (4.8), so that we decompose this as

$$
\sum_{A^{\prime} \subset D ; x \notin A^{\prime}} \sum_{\boldsymbol{\sigma}_{A^{\prime}}^{\prime}} \sum_{B_{\uparrow}^{\prime}, B_{\downarrow}^{\prime} \subset P} \cdots+\sum_{A^{\prime} \subset D ; x \in A^{\prime}} \sum_{\boldsymbol{\sigma}_{A^{\prime}}^{\prime}} \sum_{B_{\uparrow}^{\prime}, B_{\downarrow}^{\prime} \subset P} \cdots
$$

and write $\Phi_{x}$ for the first sum. Since the states in the first sum and those in the second sum are linearly independent, $\Phi_{x}=0$ must be satisfied.

To obtain $\Phi_{x}$ for $\sigma=\uparrow$, we operate $\mathcal{P}_{D} d_{x, \downarrow}^{\dagger} b_{x, \uparrow} \mathcal{P}_{D}$ on the basis state $\mathcal{P}_{D} \Phi\left(A, \boldsymbol{\sigma}_{A} ; B, B\right)$. Then, we have

$$
\left(1-n_{x, \downarrow}\right) \mathcal{P}_{D} d_{x, \downarrow}^{\dagger} c_{x, \uparrow} \Phi\left(A, \boldsymbol{\sigma}_{A} ; B, B\right)+\beta \mathcal{P}_{D} d_{x, \downarrow}^{\dagger}\left(\sum_{u \in P_{x}} c_{u, \uparrow}\right) \Phi\left(A, \boldsymbol{\sigma}_{A} ; B, B\right)
$$

The second term in (4.21) becomes

$$
\beta \sum_{u, u^{\prime} \in P_{x}} \operatorname{sgn}\left[u, u^{\prime} ; B\right] \chi[u \in B] \chi\left[u^{\prime} \notin B\right] \mathrm{e}^{2 \mathrm{i} Q \cdot u^{\prime}} \mathcal{P}_{D} \Phi\left(A, \boldsymbol{\sigma}_{A} ; B \backslash\{u\}, B \cup\left\{u^{\prime}\right\}\right),
$$

where $\operatorname{sgn}\left[u, u^{\prime} ; B\right]$ is a sign factor arising from exchanges of fermion operators. Thus, the second term contributes to $\Phi_{x}$ only when $x \notin A$. The first term in (4.21) becomes

$$
\begin{aligned}
& \left(\chi[x \in A] \chi\left[\sigma_{x}=\uparrow\right]+\chi[x \notin A]\right) \mathcal{P}_{D} d_{x, \downarrow}^{\dagger} c_{x, \uparrow} \Phi\left(A, \boldsymbol{\sigma}_{A} ; B, B\right) \\
& \quad-\left(\chi[x \in A] \chi\left[\sigma_{x}=\uparrow\right]+\chi[x \notin A]\right) \mathcal{P}_{D} d_{x, \downarrow}^{\dagger} c_{x, \downarrow}^{\dagger} c_{x, \downarrow} c_{x, \uparrow} \Phi\left(A, \boldsymbol{\sigma}_{A} ; B, B\right) .
\end{aligned}
$$

Since site $x$ is always occupied by an electron in the second term in (4.23), this term never contributes to $\Phi_{x}$. The first term in (4.23) furthermore becomes

$$
\begin{aligned}
& \chi[x \in A] \chi\left[\sigma_{x}=\uparrow\right] \operatorname{sgn}[x ; A] \\
& \sum_{u^{\prime} \in P_{x}} \operatorname{sgn}\left[u^{\prime} ; B\right] \chi\left[u^{\prime} \notin B\right] \\
& \times \mathrm{e}^{2 \mathrm{i} Q \cdot u^{\prime}} \mathcal{P}_{D} \Phi\left(A \backslash\{x\}, \boldsymbol{\sigma}_{A \backslash\{x\}} ; B, B \cup\left\{u^{\prime}\right\}\right) \\
&+\chi[x \in A] \chi\left[\sigma_{x}=\uparrow\right] \mathcal{P}_{D}\left(\prod_{x \in A} c_{x, \sigma_{x}}^{\dagger}\right) d_{x, \downarrow}^{\dagger} c_{x, \uparrow}\left(\prod_{u \in B_{\uparrow}} a_{u, \uparrow}^{\dagger}\right)\left(\prod_{u \in B_{\downarrow}} a_{u, \downarrow}^{\dagger}\right) \\
&+\chi[x \notin A] \alpha \sum_{u, u^{\prime} \in P_{x}} \operatorname{sgn}\left[u, u^{\prime} ; B\right] \chi[u \in B] \chi\left[u^{\prime} \notin B\right] \\
& \times \mathrm{e}^{2 \mathrm{i} Q \cdot u^{\prime}} \mathcal{P}_{D} \Phi\left(A, \boldsymbol{\sigma}_{A} ; B \backslash\{u\}, B \cup\left\{u^{\prime}\right\}\right),
\end{aligned}
$$


where $\mathbf{s g n}[x ; A]$ and $\mathbf{s g n}[u ; B]$ are again sign factors. Since site $x$ is always occupied by an electron in the second term in the above expression, this term does not contribute to $\Phi_{x}$. Therefore we finally obtain

$$
\begin{aligned}
\Phi_{x}= & \sum_{A \subset D ; x \in A} \sum_{\boldsymbol{\sigma}_{A} ; \sigma_{x}=\uparrow} \sum_{B \subset P} \operatorname{sgn}[x ; A] \sum_{u^{\prime} \in P_{x}} \operatorname{sgn}\left[u^{\prime} ; B\right] \chi\left[u^{\prime} \notin B\right] \\
& \times \mathrm{e}^{2 \mathrm{i} Q \cdot u^{\prime}} \phi\left(A, \boldsymbol{\sigma}_{A} ; B\right) \mathcal{P}_{D} \Phi\left(A \backslash\{x\}, \boldsymbol{\sigma}_{A \backslash\{x\}} ; B, B \cup\left\{u^{\prime}\right\}\right) \\
+(\alpha+\beta) & \sum_{A \subset D ; x \notin A} \sum_{\boldsymbol{\sigma}_{A}} \sum_{B \subset P} \sum_{u, u^{\prime} \in P_{x}} \operatorname{sgn}\left[u, u^{\prime} ; B\right] \chi[u \in B] \chi\left[u^{\prime} \notin B\right] \\
& \times \mathrm{e}^{2 \mathrm{i} Q \cdot u^{\prime}} \phi\left(A, \boldsymbol{\sigma}_{A} ; B\right) \mathcal{P}_{D} \Phi\left(A, \boldsymbol{\sigma}_{A} ; B \backslash\{u\}, B \cup\left\{u^{\prime}\right\}\right) .
\end{aligned}
$$

It is noted that the terms in the first sum are linearly independent of those in the second sum.

Choose a configuration $\left(A, \boldsymbol{\sigma}_{A} ; B\right)$ satisfying that $A$ contains $x, \sigma_{x}$ in $\boldsymbol{\sigma}_{A}$ is $\uparrow$ and there exists $u^{\prime} \in P_{x}$ such that $u^{\prime} \notin B$. Then, by checking the coefficient of the basis state $\mathcal{P}_{D} \Phi\left(A \backslash\{x\}, \boldsymbol{\sigma}_{A \backslash\{x\}} ; B, B \cup\left\{u^{\prime}\right\}\right)$ in (4.25), we obtain $\phi\left(A, \boldsymbol{\sigma}_{A} ; B\right)=0$ for such configuration. Since this and a similar result for $\sigma=\downarrow$ must hold for any $x \in D$, we obtain that

$$
\phi\left(A, \boldsymbol{\sigma}_{A} ; B\right)=0
$$

if $\left(\cup_{x \in A} P_{x}\right)$ is not a subset of $B$.

Next, choose a configuration satisfying that $A$ does not contain $x$ and there exist $u, u^{\prime} \in P_{x}$ such that $u \in B$ and $u^{\prime} \notin B$. For this kind of configuration, by checking the coefficient of the basis state $\mathcal{P}_{D} \Phi\left(A, \boldsymbol{\sigma}_{A} ; B \backslash\{u\}, B \cup\left\{u^{\prime}\right\}\right)$ in (4.25), we have that

$$
\begin{aligned}
& (\alpha+\beta)\left(\operatorname{sgn}\left[u, u^{\prime} ; B\right] \mathrm{e}^{2 \mathrm{i} Q \cdot u^{\prime}} \phi\left(A, \boldsymbol{\sigma}_{A} ; B\right)\right. \\
& \left.\quad+\operatorname{sgn}\left[u^{\prime}, u ; B_{u \rightarrow u^{\prime}}\right] \mathrm{e}^{2 \mathrm{i} Q \cdot u} \phi\left(A, \boldsymbol{\sigma}_{A} ; B_{u \rightarrow u^{\prime}}\right)\right)=0
\end{aligned}
$$

where $B_{u \rightarrow u^{\prime}}=(B \backslash\{u\}) \cup\left\{u^{\prime}\right\}$. Since $\operatorname{sgn}[\cdot], \mathrm{e}^{2 \mathrm{i} Q \cdot u}$ and $\mathrm{e}^{2 \mathrm{i} Q \cdot u^{\prime}}$ give only sign factors and $\alpha+\beta$ is non-zero by definition, we obtain the (necessary) condition that, for any $A$ not containing $x$ and any $\boldsymbol{\sigma}_{A}$,

$$
\left|\phi\left(A, \boldsymbol{\sigma}_{A} ; B\right)\right|=\left|\phi\left(A, \boldsymbol{\sigma}_{A} ; B_{u \rightarrow u^{\prime}}\right)\right|
$$

if there exist $u, u^{\prime} \in P_{x}$ such that $u \in B$ and $u^{\prime} \notin B$.

By using conditions (4.26) and (4.28), we shall prove that $\phi\left(A, \boldsymbol{\sigma}_{A} ; B\right)=0$ for any $A \neq \emptyset$. Suppose that $\phi\left(A, \boldsymbol{\sigma}_{A} ; B\right) \neq 0$ for some non-empty set $A \subset D$, spin configuration $\boldsymbol{\sigma}_{A}$ and $B \subset P$. Here we say that $u$ and $u^{\prime}$ in $B$ are connected when $D_{u} \cap D_{u^{\prime}} \neq \emptyset$ and decompose $B$ into connected components as $B=B^{1} \cup \cdots B^{m}$. We find from condition (4.26) that $A$ is contained in $\cup_{u \in B} D_{u}$, and without loss of generality, we can assume that $\cup_{u \in B^{1}} D_{u}$ contains at least one site in $A$. For connected subset $B^{1}$ we can find at least one pair of sites $u_{0}, u_{1} \in P$ and a site $x_{1} \in \cup_{u \in B^{1}} D_{u}$ such that $u_{0} \notin B^{1}, u_{1} \in B^{1}$ and $u_{0}, u_{1} \in P_{x_{1}}$. When $m>1$ this 
is trivial, and when $m=1$ this follows from $N_{\mathrm{e}}=|A|+2|B| \leq 2|P|$. It is noted that $x_{1}$ is not in $A$ due to condition (4.26). Then, since we can always find subsets $\left\{u_{2}, \ldots, u_{n}\right\} \subset B^{1}$ and $\left\{x_{2}, \ldots, x_{n}\right\} \subset \cup_{u \in B^{1}} D_{u}$ satisfying that $u_{l} \notin B_{u_{l} \rightarrow u_{0}}^{1}, u_{l+1} \in B_{u_{l} \rightarrow u_{0}}^{1}, u_{l}, u_{l+1} \in P_{x_{l+1}}$ and $x_{l} \notin A$ for $1 \leq l<n$, and $x_{n} \in A$, the repeated use of (4.28) gives

$$
\left|\phi\left(A, \boldsymbol{\sigma}_{A} ; B\right)\right|=\left|\phi\left(A, \boldsymbol{\sigma}_{A} ; B_{u_{n-1} \rightarrow u_{0}}\right)\right| .
$$

But, since $u_{n-1} \in P_{x_{n}}$ is not in $B_{u_{n-1} \rightarrow u_{0}}$, the coefficient $\phi\left(A, \boldsymbol{\sigma}_{A} ; B_{u_{n-1} \rightarrow u_{0}}\right)$ must be vanishing because of (4.26), which leads to a contradiction. Therefore the claim is proved.

From the above results we conclude that $\Phi$ is expanded as

$$
\Phi=\sum_{B \subset P} \phi(B) \mathcal{P}_{D} \Phi\left(\emptyset, \boldsymbol{\sigma}_{\emptyset} ; B, B\right)
$$

where $\phi(B)=\phi\left(\emptyset, \boldsymbol{\sigma}_{\emptyset} ; B\right)$. Then, using (4.28) again, we find that $|\phi(B)|=\left|\phi\left(B^{\prime}\right)\right|$ whenever $|B|=\left|B^{\prime}\right|$, which completes the proof of Lemma 4.1

\section{Acknowledgement}

I would like to thank Kengo Tanaka for many discussions.

\section{References}

[1] P. W. Anderson, Science 235, 1196 (1987).

[2] U. Brandt and A. Giesekus, Phys. Rev. Lett. 68, 2648 (1992).

[3] H. Tasaki, Phys. Rev. Lett. 70, 3303 (1993).

[4] P.-A. Bares and P. A. Lee, Phys. Rev. B 49, 8882 (1994).

[5] M. Yamanaka, S. Honjo, Y. Hatsugai and M. Kohmoto, J. Stat. Phys. 84, 1133 (1996).

[6] C. N. Yang, Phys. Rev. Lett. 63, 2144 (1989).

[7] F. H. L. Essler, V. E. Korepin and K. Schoutens, Phys. Rev. Lett. 70, 73 (1993).

[8] J. de Boer, V. E. Korepin and A. Schadschneider, Phys. Rev. Lett. 74, 789 (1995).

[9] I. N. Karnaukhov and A. A. Ovchinnikov, Phys. Rev. B 66, 104518 (2002).

[10] X.-Y. Ge and M. D. Gould, J. Phys. A: Math. Gen. 36, 1801 (2003). 
[11] S.-Q. Shen and Z.-M. Qiu, Phys. Rev. Lett. 71, 4238 (1993).

[12] S.-Q. Shen, Phys. Rev. B 54, 9039 (1996).

[13] A. Lanzara et al., Nature 412, 510 (2001).

[14] H. Tasaki, Phys. Rev. B 49, 7763 (1994).

[15] T. Koma and H. Tasaki, J. Stat. Phys. 76, 745 (1994). 